\title{
Catégorisation sociopolitique et hors-groupe
}

\author{
L'apport d'une approche fonctionnaliste
}

Nicolas Mary

\begin{abstract}
Cet article présente quelques éléments de réflexion sur la catégorisation sociopolitique et la construction d'hors-groupes, c'est-à-dire de groupes faisant l'objet d'une certaine hostilité pouvant engendrer des injustices sociales. Nous nous proposons de montrer que la mise à l'écart de l'hors-groupe précède l'élaboration des stéréotypes négatifs qui sont évoqués pour la justifier. Ceci nous conduira à envisager l'hors-groupe dans sa dimension fonctionnelle, dont la prise en compte seule permettrait de lutter efficacement contre les discriminations subies.

Mots-clefs : catégorisation, hors-groupe, fonctionnalisme, Merton

This article submits some insights on sociopolitical categorization and the making of outgroups - that is, groups facing hostility and subsequent social injustices. We suggest that the sidelining of out-groups precedes the elaboration of the negative stereotypes which are thereafter evoked to justify it. Such a stance will drive us to consider the outgroup in its functional dimension, which may prove useful in addressing discriminations.
\end{abstract}

Keywords: categorization, outgroup, functionalism, Merton

\section{Introduction}

Dans son ouvrage intitulé Social Theory and Social Structure (1949), Robert King Merton proposait un paradigme de l'analyse fonctionnelle qui ne devait pas, selon lui, s'appliquer à l'ensemble du monde social, mais seulement à certains éléments standardisés, c'est-à-dire appelés à se répéter. Le but de cet article est de décrire ce qu'une telle approche pourrait encore apporter à un débat sur les catégories habituellement alimenté par la psychologie sociale.

Nous verrons dans une première partie qu'en construisant une représentation de l'environnement social en termes de groupes hiérarchisés, la catégorisation aboutit à l'identification de ce que nous appellerons des hors-groupes, qui font l'objet d'une hostilité plus ou moins marquée pouvant engendrer des injustices sociales. Nous nous efforcerons ensuite de montrer que si les stéréotypes négatifs associés à l'hors-groupe 
semblent devoir résister à toute entreprise de réfutation, c'est qu'ils ne constituent pas la cause de son rejet, mais sa justification a posteriori. Nous essaierons alors d'identifier les fonctions de l'hors-groupe qui en expliquent la pérennité, avant d'évoquer ce qui pourrait constituer des équivalents fonctionnels à sa stigmatisation et dont il conviendrait en conséquence de favoriser l'émergence.

\section{Cadre théorique}

Avant d'aller plus loin, quelques précisions sémantiques s'imposent. Il nous faut, en premier lieu, préciser ce que nous entendons par « groupe ». «Le concept sociologique de groupe définit un ensemble d'individus en interaction selon des règles établies ou encore un certain nombre de gens ayant entre eux des rapports sociaux caractérisés et fixés » (MERTON 1968, p.240). À ce critère objectif, l'interaction, le sociologue en ajoute deux autres, subjectifs ceux-là : les individus en interaction doivent se considérer euxmêmes comme membres du groupe, ils doivent également être reconnus comme tels par les autres, membres et non-membres. Le terme de groupe, ainsi défini, ne saurait être utilisé pour désigner un grand nombre de gens car le critère d'interaction ne serait pas respecté. Dans ces conditions, il ne pourrait être question de groupe national ou de groupe ethnique. Cette notion doit donc être étirée pour nous être utile. Ainsi, par groupe, non entendrons un ensemble d'individus en interaction " potentielle » selon des règles établies; chacun de ces individus se reconnaissant et étant reconnu comme membre du groupe.

Le terme « d'hors-groupe » pour désigner un groupe qui suscite une certaine hostilité est quant à lui emprunté à William Graham Sumner.

«La notion de "société primitive" qu'il nous faut adopter renvoie à des petits groupes éparpillés sur un territoire [...]. Des groupes peuvent nouer des relations (parenté, voisinage, alliance, mariage, commerce) qui les rapprochent les uns des autres et les séparent d'autres groupes. Une différence naît alors entre Nous, l'engroupe, et tous les autres, les hors-groupes. [...] Au sein de l'en-groupe, les relations sont fondées sur les notions de paix, ordre, loi, gouvernement et travail. Les relations avec les hors-groupes sont basées sur la guerre et le pillage, excepté lorsque des accords ont été conclus qui les interdisent. »(SUMNER 2002, paragraphe 13)

Ces concepts d'en-groupe et d'hors-groupe semblent pertinents pour étudier les mécanismes de discrimination dont peuvent être victimes certaines catégories sociopolitiques. Il ne faut bien se garder cependant, de tenir les notions d'en-groupe et de groupe d'appartenance (ou endogroupe) pour équivalentes, pas plus du reste, que celles d'hors-groupe et de groupe de non-appartenance (ou exogroupe). En effet, et contrairement à ce que semblait penser William Sumner, l'allégeance à un groupe est loin d'impliquer nécessairement l'hostilité envers les autres et du point de vue de leurs membres, tous les groupes d'appartenance ne sont pas des en-groupes. La théorie du groupe de référence montre bien qu'un groupe de non-appartenance peut être perçu 
d'une manière très favorable ${ }^{1}$. Il faut nous donc envisager en-groupe et hors-groupe comme des sous-groupes inclus dans une organisation sociale plus ample.

En résumé, au sein d'un groupe d'appartenance peuvent se former des en-groupes, caractérisés par une forte cohérence interne et une hostilité plus ou moins marquée à l'égard de groupes de non-appartenance, perçus comme des hors-groupes. Ainsi, par exemple, à l'intérieur d'un groupe ethnique, existerait un en-groupe raciste, à l'intérieur d'un groupe politique quelconque, existerait un en-groupe sectaire, etc.

\subsection{Les vertus de l'en-groupe et les vices de l'hors-groupe}

Le rejet de l'hors-groupe est justifié non pas, à proprement parler, par sa différence, qui peut reposer sur des fondements objectifs comme l'origine ethnique ou la religion, mais par un stéréotype négatif qui lui est associé2. On ne va pas faire grief à l'horsgroupe de ce qui peut constituer le fondement de son identité, mais on va lui attribuer certains «vices». Or, si on ne saurait lutter efficacement contre les discriminations subies par l'hors-groupe en s'efforçant de démontrer le caractère erroné des préjugés qui s'y attachent, c'est que, contrairement à ce que l'on a coutume de penser, ceux-ci sont davantage le symptôme que la raison de cette mise à l'index. Ils ne sont pas la cause de l'ostracisme, mais ils en constituent une justification a posteriori.

Ceci peut-être déduit des travaux de Robert King Merton relatifs aux antagonismes ethniques qui minaient la société américaine, et ce bien que lui-même n’ait pas été jusque là. Le sociologue avait en effet relevé que le rejet des minorités ethniques par la middle class blanche dominante perdurait quoi qu'elles puissent faire. Selon lui, «les hors groupes ethniques [étaient] maudits, qu'ils adoptent ou non les valeurs de la société blanche protestante » (MERTON 1968, p.147). Pour illustrer son propos, Robert King Merton s'appuyait sur l'exemple d'Abraham Lincoln comme incarnation des valeurs de la middle class blanche américaine : «Le moindre écolier sait que Lincoln était économe, laborieux, désireux d'apprendre, ambitieux, respectueux des droits du citoyen moyen, et particulièrement habile à escalader l'échelle de la chance depuis l'échelon le plus bas, celui de manœuvre, jusqu'aux sommets enviés où planent le marchand et l'avocat. » (MERTON 1968, p.148).

\footnotetext{
${ }^{1}$ Nous pouvons, pour illustrer, évoquer ce que l'économiste James Duesenberry a appelé « l'effet d'imitation»- le fait qu'un groupe social donné a tendance à imiter les habitudes de consommation d'un groupe jugé supérieur.

2 C'est-à-dire un ensemble de croyances et de jugements qui s'y réfèrent. Par exemple, on ne reprochera pas, ou rarement, aux Juifs de nier le dogme de la Trinité, mais par contre on va les trouver avares, fourbes, etc.
} 


\subsection{La prophétie auto réalisatrice}

En conséquence, nous pouvons convenir qu'il est, d'une certaine manière, parfaitement logique que cette middle class américaine méprise et rejette ceux qu'elle considère étrangers à ces vertus. On conçoit ainsi que l'en-groupe blanc américain ait tendance à mépriser les Noirs parce que, comme le veut le stéréotype, ils ne sont pas économes, ils ne sont pas ambitieux, ils ne veulent pas apprendre, etc. Ce rejet s'appuie même sur des éléments a priori objectifs puisque certaines études statistiques vont apparemment dans ce sens, en révélant par exemple que les Noirs ont un niveau moyen d'instruction plus bas, sont sous représentés dans les professions commerciales et libérales, etc.

Ces accusations sont en fait profondément injustes dans la mesure où les Noirs américains sont largement victimes de ce que Robert King Merton a baptisé la prophétie auto réalisatrice («self-fulfilling prophecy»). Celle-ci part de l'analyse spécieuse d'une situation, qui, parce qu'elle est admise comme une réalité objective, provoque une modification des comportements qui rend vrai ce qui ne l'était pas à l'origine (MERTON 1968, p.143). Ainsi, selon Merton, parce que l'en-groupe blanc dominant tient les Noirs pour intellectuellement inférieurs, il se refuse à gaspiller pour eux le budget de l'éducation et se justifiera en arguant du fait qu'ils comptent cinq fois moins de diplômés d'université que les Blancs, inégalité qu'il aura lui-même perpétuée.

Ici pourtant, le mécanisme est sans doute beaucoup plus complexe que le suppose le sociologue. Il est en effet relativement facile de justifier l'argent dépensé pour les Blancs sans conclure à une injustice. Le niveau d'instruction n'est pas seulement une question d'argent. Un membre de l'en-groupe blanc pourrait ainsi aisément soutenir que si l'on dépense cinq fois plus d'argent pour un écolier blanc, c'est que celui-ci reste plus longtemps dans le système scolaire parce qu'il est plus «désireux d'apprendre » et intellectuellement supérieur! La prédiction auto réalisatrice agit donc d'une manière qui n'est pas aussi évidente que le dit Robert Merton.

En effet, il faut déjà considérer que les hors-groupes sont enclins à se conformer à l'image stéréotypée que leur renvoie d'eux-mêmes le groupe dominant. De ce point de vue, si les écoliers noirs ont, en moyenne, de moins bons résultats, c'est que certains ont tendance à moins s'investir parce que leur a été inculquée l'idée que les études n'étaient pas faites pour eux³ ${ }^{3}$ À cela s'ajoute encore un effet Pygmalion, qui fait par exemple que, parce que leurs maitres ont admis une fois pour toute que les Noirs étaient moins bons élèves, ils vont se montrer moins exigeants avec eux, ne les incitant pas ainsi à exploiter tout leur potentiel. De plus, même lorsque que le stéréotype négatif

\footnotetext{
${ }^{3}$ Ce même mécanisme aide aussi à expliquer la sous-représentation des filles dans les filières
} scientifiques. 
n’a pas été intériorisé par le membre de l'hors-groupe, il peut avoir une incidence sur ses performances. Ainsi, Claude Steele et Joshua Aronson ont-ils établi que «la menace du stéréotype », c'est-à-dire la possibilité d'être jugé en fonction d'un stéréotype négatif, peut induire une pression supplémentaire (surcroît d'anxiété, lenteur par peur de mal faire, etc.) qui vient interférer avec les performances (STEELE \& ARONSON 1995). Il faut enfin considérer un dernier élément qui concoure à vicier les statistiques. En effet, dès lors que l'écolier noir prend, comme groupe de référence, son groupe d'appartenance, dont les performances scolaires sont moins bonnes ${ }^{4}$, il aura tendance à être moins ambitieux, contribuant de la sorte à perpétuer une inégalité dont il est victime ${ }^{5}$.

Ainsi, si le fait que les hors-groupes soient condamnés parce qu'ils ne font pas preuve d'une adhésion aux valeurs du groupe dominant n'a pas vraiment de quoi surprendre tant cette conception relève d'un ethnocentrisme assez banal: il faut en revanche considérer le rôle de la prophétie auto-réalisatrice qui empêche l'hors-groupe de sortir du stéréotype négatif dans lequel on entend l'enfermer.

\subsection{Une alchimie morale}

«Avoir du talent ne suffit pas : il faut aussi avoir votre permission d'en avoir - eh quoi mes amis ?»

Friedrich Nietzsche, Par delà le bien et le mal.

On s'étonnera certainement davantage en constatant que la middle class blanche américaine rejette tout aussi surement les hors-groupes s'ils démontrent les vertus qu'elle prône ${ }^{6}$. En effet, dès lors que c'est le membre d'un hors-groupe qui montre les qualités célébrées par le groupe dominant, intervient une « alchimie morale » qui permet à l'en-groupe de transmuter à loisir vice en vertu et vertu en vice. La mise à l'écart subie par l'hors-groupe ne dépend donc pas de ce qu'il fait puisque qu'un même comportement prend une valeur différente lorsqu'il passe de l'en-groupe à l'horsgroupe.

C’est un phénomène qui avait déjà été observé chez les Trobriandais par Bronislaw Malinowski. Chez les Argonautes du Pacifique en effet, les succès féminins confèrent, en règle générale, honneur et prestige. Mais qu'un Trobriandais du commun ait auprès des

\footnotetext{
${ }^{4}$ Quelles qu'en soient les raisons.

${ }^{5}$ Une autre réaction est possible, c'est monter en épingle les succès des Noirs, d'où une image d'arrogance qui vient encore justifier l'exclusion.

${ }^{6}$ Le stéréotype associé à l'hors-groupe reste négatif, même s'il correspond en fait aux valeurs célébrées par le groupe dominant.
} 
dames, «trop» de succès, réussite qui doit être réservée à l'élite, et ses conquêtes deviennent un objet de scandale. Les chefs s'indignent ainsi facilement d'un pouvoir de séduction qui n’est pas justifié par la position sociale. «Une activité louable, commente Robert Merton, lorsqu'elle est remplie par des gens qui n'y sont pas destinés, devient un objet de mépris et non d'honneur. Car, c'est seulement en gardant le monopole de ces vertus que les hommes en place pourront conserver leurs privilèges, leur prestige et leurs pouvoirs. On ne saurait imaginer meilleur moyen de préserver un système de pouvoir et de stratification sociale» (MERTON 1968, p.142). Les vertus ne restent donc des vertus que dès lors qu'elles restent l'apanage du groupe dominant. Qu'elles soient démontrées par qui n'y est pas socialement autorisé et les voilà transmutées en vices par un subtil glissement sémantique. Ainsi, lorsque Lincoln travaille dans la nuit, c'est le signe qu'il est industrieux, résolu, persévérant et volontaire. Mais qu'un hors-groupe juif ou japonais fasse de même et il témoigne lui d'une mentalité de "sweat shop", d’un "grignotage obstiné des standards américains", il fait preuve d'une concurrence déloyale. De même, si Lincoln est frugal, travailleur et économe, Cohen, lui, est avare, ladre et grippe-sous. Pour s'élever, Lincoln s'est montré débrouillard, habile et intelligent, alors que l'hors-groupe est combinard, arriviste et fourbe, etc?

L'anathème est donc jeté sur les membres de l'hors-groupe, qu'ils pratiquent ou non les vertus prônées par l'en-groupe. C'est pour cette raison qu'il est définitivement vain d'espérer en finir avec le rejet d'un hors-groupe en démontrant que le stéréotype négatif qu'on lui associe est faux. Cela revient en fait à se comporter comme l'agneau de la fable. Quand bien même parviendrait-on à détruire un préjugé qu'il serait immédiatement remplacé par un autre, car il faudrait toujours justifier l'exclusion de l'hors-groupe.

\section{Une approche fonctionnelle de l'hors groupe}

Ainsi, il nous est permis de supposer que si le rejet de l'hors-groupe perdure malgré la spéciosité parfois évidente des arguments qui viennent le justifier, c'est que l'horsgroupe peut se révéler fonctionnel pour le groupe ou l'en-groupe. Il nous reste à comprendre en quoi.

${ }^{7}$ Il faut se garder cependant de voir dans cette conversion des vertus du groupe dominant ou de l'en-groupe en vices de l'hors-groupe un complot destiné à pérenniser l'existence de ce dernier. L'indignation suscitée par les réussites de l'hors-groupe, pour être moralement condamnable, n'en est pas moins parfaitement spontanée et sincère. 


\subsection{Un bouc émissaire désigné}

Un premier élément de réponse nous est suggéré par Emile Durkheim dans Antisémitisme et crise sociale: "Quand la société souffre, elle éprouve le besoin de trouver quelqu'un à qui elle puisse imputer son mal, sur qui elle se venge de ses déceptions ; et ceux-là sont naturellement désignés pour ce rôle auxquels s'attache déjà quelque défaveur de l'opinion» (DuRKHEIM 2007, p.32). Ainsi, l'existence d'un horsgroupe serait-elle nécessaire pour servir le cas échéant de bouc émissaire, de victime expiatoire. Il nous faut, à ce titre, remarquer que c'est avec une intensité variable que les hors-groupes sont exposés à la vindicte de l'en-groupe et que les périodes de crise semblent effectivement particulièrement propices aux déchaînements. L'anathème jeté sur l'hors-groupe placerait donc l'en-groupe en meilleure position pour les affronter.

Et Durkheim de décrire la façon dont a été accueilli le verdict dans l'affaire Dreyfus : «Ce fut un élan de joie sur les boulevards [...]. On savait enfin à qui s'en prendre du trouble économique et de la détresse morale où l'on vivait! C'est des juifs que venait le mal. Le fait était officiellement constaté. Par cela seul, il semblait que tout allait mieux et on se sentait comme réconforté » (DURKHEIM 2007). Ainsi, comme il le laisse entendre, l'hors-groupe aurait une fonction explicative. Sa stigmatisation trouverait son origine dans le désarroi de ceux qui subissent des situations malheureuses qui les dépassent largement, soit par leur complexité, soit aussi parce qu'ils n'ont aucune prise sur elles. Aussi vont-ils chercher refuge dans le confort d'un univers dont la schématisation, extrême, en plus de donner une illusion de cohérence aux événements auxquels ils sont confrontés, les guérit de leur sentiment d'impuissance en leur indiquant une conduite, puisque désormais l'ennemi est identifié.

En fait, l'hors-groupe remplit ici une fonction très similaire à celle de la sorcellerie chez les Azandé, qui, comme l'indique Evans-Pritchard, fournit à la fois «une philosophie naturelle qui explique les rapports des hommes et des éléments malencontreux» et «un moyen tout prêt et tout classique de réagir à pareils événements» (EVANS-PRITCHARD 1972, p.96). Ainsi, lorsque par exemple un chasseur est tué par un éléphant, c'est qu'il a été ensorcelé. Non pas que les Azandé ignorent la causalité naturelle. L'éléphant n'est pas pour eux une créature née de la sorcellerie. Mais ils pensent que jamais l'accident ne se serait produit si un maléfice n'avait été à l'œuvre ${ }^{8}$. Une métaphore de la chasse leur permet d'exprimer très clairement cette façon de penser. En effet, lorsqu'un gibier est tué, l'usage veut que sa viande soit partagée entre le chasseur qui le premier a atteint l'animal de sa lance et celui qui l'a touché en second,

${ }^{8}$ De même que dans nos sociétés, ceux qui attribuent leurs malheurs aux agissements plus ou moins occultes de hors-groupes, peuvent aussi en même temps se montrer tout à fait rationnels dans l'analyse de leur situation. 
qu'on appelle «l'umbaya » (la seconde lance). Les Azandé considèrent que ce sont les deux hommes qui ont tué la bête. De même, lorsqu'un chasseur est écrasé par un éléphant, ils voient le pachyderme comme la première lance et la sorcellerie comme la seconde. C'est pourtant cette dernière qui est toujours privilégiée dans leurs explications car c'est elle qui indique une conduite. Si seul l'animal est en cause, il n'y a rien à faire. Alors que la sorcellerie, elle, appelle la vengeance' !

\subsection{Renforcer la cohésion de l'en-groupe}

Cette stigmatisation de l'hors-groupe est encore une façon de renforcer la cohésion du groupe, ou, à défaut, de l'en-groupe. C'est du reste pour cette raison aussi qu'il est souvent agité comme une menace.

Ainsi, dans l'Allemagne des années trente, les déchainements contre les hors-groupes français et juifs ont pu agir comme le ferment d'une unité nationale encore récente et mise à mal par la défaite de 1918 et les troubles qui ont accompagné la naissance de la République de Weimar. C'est un mécanisme qu'avait déjà su exploiter le chancelier Otto Von Bismarck qui, en 1870, avait jugé opportun, pour sceller une unité allemande obtenue à coups de canon, de détourner sur l'hors-groupe français les sentiments antiprussiens qui pouvaient subsister au sein de sa jeune nation ${ }^{10}$.

Le grand homme d'Etat eut une sorte d'héritier en la personne d'Edouard Drumont, auteur en 1886 de La France Juive, qui espérait que l'antisémitisme pourrait réunir sous sa bannière classes populaires et conservateurs catholiques. Le journaliste attendait le prince chrétien qui résoudrait la question sociale en confisquant les biens des Juifs. Sa tentative se solda néanmoins par un échec; les conservateurs se méfiaient en effet un d'anticapitalisme juif qui risquait de s'étendre au capitalisme catholique. Ils ont en revanche très vite vu le bénéfice qu'ils pouvaient tirer d'un antisémitisme débarrassé des accents socialisants que lui avait donnés Drumont.

«Dès lors qu’on désigne aux yeux des foules indigentes, aux petits commerçants et aux artisans victimes de l'évolution économique, aux ouvriers exploités et aux

\footnotetext{
${ }^{9}$ Du reste, elle n'est jamais évoquée que dans des cas que nous attribuerions nous à la fatalité. Il n'en est jamais question lorsque l'on sait qui est responsable d'un malheur. Celui qui est pris en flagrant délit d'adultère ou de mensonge, par exemple, ne pourra pas se défendre en disant avoir été ensorcelé.

${ }^{10}$ De ce point de vue, la stigmatisation de l'hors-groupe, si elle renforce la cohésion de certains groupes, peut aussi s'avérer dysfonctionnelle pour la société prise dans son ensemble. Ainsi, au début des années 90, les Serbes ont peut-être été rassemblés par la dénonciation des hors-groupes croates et musulmans, mais cela a finalement conduit la société yougoslave à l'éclatement. De même, au moment de l'affaire Dreyfus, l'antisémitisme a fragilisé la cohésion nationale de la France.
} 
paysans contraints à l'exode rural, les juifs responsables de tous leurs maux, on offre une arme inestimable à la conservation sociale qui, forte de son contrôle sur la presse, va orchestrer le développement du mythe à son propre usage. » (WINOCK 1982 :137)

La stigmatisation de l'hors-groupe pourrait donc, dans certains cas, agir comme un ferment de conservation sociale - ou, si l'on préfère, de stabilité - en étouffant les conflits de classe. L'efficacité d'un procédé aussi grossier a de quoi interpeller. Pour la comprendre, il convient de faire un détour par la théorie de «l'identité sociale » d'Henri Tajfel et John Turner (TAJFEL 1981).

\subsection{Un faire valoir}

« Moins le blanc est intelligent, plus le noir lui parait bête » André Gide, Voyage au Congo, 1927.

L'identité sociale est la partie du concept de soi d'un individu qui résulte de la conscience qu'il peut avoir d'appartenir à des groupes et de la valeur émotionnelle qu'il y attache. En conséquence, une façon de renforcer son estime de soi peut être de se construire une identité sociale positive ${ }^{11}$. Or, celle-ci dépend de la valeur des groupes impliqués dans son élaboration, cette valeur étant déterminée par comparaison avec d'autres groupes selon certains critères. C'est ainsi pour maintenir ou acquérir une certaine supériorité par rapport aux autres que les groupes sont amenés à se différencier et à adopter des comportements discriminatoires.

Les conflits intergroupes ne trouvent donc pas seulement leur origine dans des intérêts matériels incompatibles. On peut aussi s'y engager afin d'obtenir plus de dignité, plus de prestige. Nous pourrions même aller plus loin et suggérer que la recherche de gains matériels n'est encore bien souvent qu'une façon d'acquérir une identité plus positive. Il faut ainsi considérer que l'enjeu des conflits de classe n'est pas simplement la répartition du pouvoir et des richesses. Les luttes ouvrières ne sont pas seulement motivées par la perspective d'une amélioration de la situation matérielle du prolétariat, souvent dérisoire au regard des efforts consentis. Leur portée est en grande partie symbolique. Elles procèdent également, et peut-être surtout, de la volonté de valoriser l'identité sociale de ceux qui s'y engagent, dans la mesure où obtenir une augmentation salariale, aussi faible soit-elle, c'est remporter une victoire qui vient conforter l'estime de soi.

Reste que pour valoriser une identité sociale, il est des alternatives aux conflits intergroupes. En effet, pour qui n'est pas satisfait de l'identité sociale que lui confère

\footnotetext{
${ }^{11} \mathrm{Il}$ convient ici de voir que l'identité ne se limite bien sûr pas à l'identité sociale.
} 
l'appartenance à un groupe, la première possibilité, et parfois la plus simple, est de rechercher une " mobilité individuelle », c'est-à-dire de se désidentifier à un groupe pour s'efforcer d'en intégrer un autre, plus valorisé. C'est cette voie qui semble privilégiée dans les sociétés contemporaines, plus individualistes, dans lesquelles émerge, selon Claude Dubar, une forme identitaire qui «suppose l'existence de collectifs multiples, variables, éphémères, auxquels les individus adhèrent pour des périodes limitées et qui leur fournissent des ressources d'identification qu'ils gèrent de manière diverse et provisoire » (DUBAR 2000, p.5).

Une autre alternative, collective celle-là, n'implique pas de remettre en cause son identification à un groupe. Il s'agit simplement de modifier ceux par rapport auxquels on s'évalue pour que la comparaison soit flatteuse. C'est dans cette perspective que les hors-groupes trouvent leur utilité. La stigmatisation qu'ils subissent, les stéréotypes négatifs qui leurs sont associés en font des parias, ce qui permet à ceux qui se comparent à eux de se forger une identité sociale plus satisfaisante. Ainsi, en désignant l'hors-groupe à la vindicte, on désigne aux groupes dominés - dont des membres pourraient en conséquence vouloir bousculer les hiérarchies par le conflit - un nouveau point de comparaison qui leur conférera une identité sociale plus positive, rendant les revendications de classe moins nécessaires. C'est la raison pour laquelle l'antisémitisme a pu agir comme un instrument de conservation sociale. C'est aussi pour cela que les couches sociales les plus basses ou celles dont la situation est fragilisée et incertaine, c'est-à-dire les catégories dont l'identité sociale des membres a le plus besoin d'être valorisée, constituent un vivier de recrutement fécond pour les en-groupes.

\section{Une fatalité ?}

Les hors-groupes existent donc parce qu'ils sont fonctionnels. Ils sont là pour éventuellement expliquer les malheurs du monde, maintenir la cohésion du groupe ou de l'en-groupe et pour assurer une certaine stabilité sociale en permettant aux membres de groupes dominés de se construire une identité sociale positive autrement que par le conflit. Si là se trouvent les causes de la discrimination, c'est en agissant à ce niveau que l'on peut espérer atténuer les injustices sociales dont sont victimes les hors-groupes. Il s'agirait donc de favoriser l'émergence d'équivalents fonctionnels qui pourraient être mobilisés à la place de la stigmatisation de l'hors-groupe.

Robert King Merton était plutôt pessimiste quant à la possibilité de supprimer les préjugés raciaux par une campagne d'éducation : «Les idées fausses ne s'évanouissent sagement au seul contact de la vérité. On ne s'attend pas à voir un paranoïaque abandonner ses lubies et ses erreurs chèrement acquises, à la seule affirmation qu'elles ne sont pas fondées » (MERTON 1968, p.145). Et le sociologue de s'interroger sur l'espérance de vie, dans le Mississipi, d'un instituteur qui s'efforcerait d'extirper de ses élèves les croyances raciales qui leur ont été inculquées par leurs familles. Sans doute at-il raison de souligner le faible impact qu'aurait une campagne d'éducation 
spécifiquement tournée vers la dénonciation des discriminations envers l’hors-groupe, puisque, comme nous l'avons dit, elle n'en agirait pas sur les causes. Il ne faut pas cependant négliger l'effet bénéfique que pourrait produire une élévation générale du niveau culturel. En effet, plus l'individu est cultivé, plus il est intellectuellement armé pour comprendre son environnement et donc moins il aura besoin de bouc émissaire pour expliquer les malheurs du monde. Il serait du reste intéressant de tester la corrélation entre la violence des déchainements contre l'hors-groupe et le niveau d'éducation.

Par ailleurs, une autre fonction de l'hors-groupe est de constituer un point de référence à partir duquel la comparaison sera flatteuse pour tous les autres groupes, ce qui valorise l'identité sociale de leurs membres. Une façon d'échapper à la condition d'hors-groupe pourrait être alors de conforter l'en-groupe dans son sentiment de supériorité autrement qu'en subissant ses foudres. Dans ce sens, une étude menée au sein de l'armée américaine durant la seconde guerre mondiale établissait par exemple que, «parmi les soldats qui considéraient en septembre 1943 que la discipline n'était pas trop stricte, $19 \%$ étaient caporaux en janvier 1944 contre 12\% pour les autres. » Pour Robert Merton, qui a commenté cette enquête, le phénomène mis en évidence est celui de la «socialisation anticipatrice », c'est-à-dire que l'individu qui adopte les valeurs du groupe auquel il souhaite appartenir augmente ses chances de succès. Ce phénomène s'explique assez aisément avec la théorie de l'identité sociale. En manifestant son envie de s'identifier à un groupe, l'individu donne à ses membres un sentiment de supériorité. Il valorise donc leur identité sociale. Ainsi, le membre d'un hors-groupe peut-il échapper à cette condition inconfortable en affichant sa volonté de se désidentifier à l'hors-groupe pour rejoindre le groupe dominant.

Enfin, une autre stratégie, collective celle-là, est de s'efforcer d'assigner à un autre groupe les fonctions d'hors-groupe. Ainsi, en France, la stigmatisation des immigrés italiens, espagnols ou polonais ${ }^{12}$ a-t-elle cessé avec l'arrivée de nouvelles migrations en provenance d'Afrique. Dans ce sens, la montée de l'antisémitisme en France au début des années 2000, dans la population musulmane issue des quartiers dits sensibles, n'est peut-être pas seulement l'expression d'une solidarité religieuse dans le conflit israélo-

12 A propos desquels Philippe Ariès (1971) écrivait par exemple: «Une véritable invasion, méthodique, où les émigrés arrivaient groupés, avec leurs prêtres, leurs instituteurs, leurs religieuses. Des cités entières leur furent affectées, qui constituent de véritables villages étrangers, où le français n'est pas compris, où les relents de cuisine rappellent les odeurs de l'Europe centrale. Cette population est composée de beaucoup d'étrangers inassimilables, qui vivent en groupes fermés, avec leurs églises, leurs écoles, leurs magasins, leurs jeux, étrangers au reste de la population » (ARIES 1971, pp.110-111). 
palestinien ${ }^{13}$. Elle n'est peut-être pas seulement non plus la manifestation du besoin de se forger une identité sociale positive. Elle est peut-être aussi le fruit d'une volonté d'échapper à la condition d'hors-groupe en désignant d'autres parias à la vindicte de l'en-groupe dominant ${ }^{14}$.

\section{Conclusion}

La catégorisation s'accompagne donc de l'identification d'hors-groupes dont l'ostracisme est justifié par les stéréotypes négatifs qui leurs sont associés. Or cette justification n'intervient qu'a posteriori. Les croyances relatives à l'hors-groupe ne sont pas la cause de sa marginalisation, elles naissent du besoin d'occulter les raisons véritables de sa mise à l'écart, de faire écran entre l'en-groupe et la réalité objective de son comportement. En fait, si des hors-groupes sont identifiés, c'est parce qu'ils remplissent une fonction explicative, ils agissent comme un ferment de cohésion et de stabilité sociale en permettant à des groupes dominés d'acquérir une identité sociale positive.

Il n'y a cependant pas de fatalité et il est d'autres moyens de faire cesser les injustices dont est victime l'hors-groupe que de les faire subir à un autre groupe. Il existe à sa mise à l'index des équivalents fonctionnels qu'il conviendrait de promouvoir. Cela ne semble pourtant pas être une direction souvent prise par les politiques gouvernementales qui auraient plutôt tendance à consolider les barrières construites autour des hors-groupes ${ }^{15}$, quand elles ne vont pas jusqu'à encourager la stigmatisation ${ }^{16}$. C'est précisément ce qui avait été reproché à Mr. Nicolas Sarkozy en 2005, lorsqu'il avait parlé de «nettoyer au kärcher» certaines cités dont les habitants étaient majoritairement issus de l'immigration ${ }^{17}$. En effet, pour les dirigeants aussi, la stigmatisation d'hors-groupes peut s'avérer un moyen bien commode de susciter une certaine adhésion tout en éludant leurs responsabilités dans les difficultés que peuvent

13 Cf. Le Monde diplomatique du 4 avril 2003, «Violences antisémites sur fond de guerre en Irak ». Le ministère de l'Intérieur avait à ce titre relevé que les actes antisémites ne concernaient pas l'ensemble de la population musulmane mais des groupes bien limités. Leurs auteurs étaient de jeunes adultes issus de quartiers « sensibles ».

${ }_{14}$ D'après le ministère de l'intérieur les auteurs des agressions antisémites identifiés se trouvaient souvent dans une situation de "marginalité sociale».

15 Ainsi, en étudiant les conditions d'attribution des logements sociaux en France et en GrandeBretagne, Valérie Sala Pala a montré de quelle façon la catégorisation ethnique pouvait se trouver au cœur même de l'action publique (SALA PALA 2005).

16 Même la discrimination positive y concoure puisque, d'une certaine manière, elle consacre l'infériorité de l'hors-groupe tout en l'exposant aux accusations de concurrence déloyale.

${ }^{17}$ Il ne s'agissait, selon Nicolas Sarkozy, que d'en éradiquer la délinquance. Mais cela allait aussi dans le sens d'une assimilation, souvent faite, de l'immigration à la délinquance. 
connaître les gouvernés. En fait, toute la difficulté vient du fait que ceux peuvent agir contre la construction d'hors-groupes ne sont pas nécessairement ceux qui ont le moins d'intérêt à leur pérennisation...

\section{Bibliographie}

ARIES P. (1971): Histoire des populations françaises [1948]. Paris: Seuil.

DUBAR C. (2000): La crise des identités, l'interprétation d'une mutation. Paris: PUF.

Durkheim E. (2007): L'individualisme et les intellectuels. Paris: Mille et une nuits.

Evans-Pritchard E. E. (1972): Sorcellerie, oracles et magie, chez les Azandé. Paris: Gallimard.

Merton R.K. (1968): Eléments de théorie et de méthode sociologique. Paris: Plon.

SAla PALA V. (2005): Politique du logement social et construction des frontières ethniques. Une comparaison franco-britannique. Thèse pour le doctorat de science politique. Rennes: université de Rennes 1.

Steele C., Aronson J. (1995): «Stereotype threat and the intellectual test performance of african Americans ». In Journal of Personality and Social Psychology, vol. $69 \mathrm{n}^{\circ} 5$, pp.797-811.

Sumner W.G. (2002): Folkways. New York: Dover Publications.

TAJFEL H. (1981): Human Groups and Social Categories. Cambridge: Cambridge University Press.

WINOCK M. (1982): Nationalisme, antisémitisme et fascisme en France. Paris: Seuil. 